Pacific Journal of Mathematic 


\title{
A SET FUNCTION DEFINED FOR CONVEX PLANE DOMAINS
}

\author{
M. I. AISSEN
}

Introduction In this note, we define a set function for bounded plane convex domains and study its properties. In particular, we exhibit its analogy to a classical set function from the theory of conformal mapping. The function is of interest because it is involved in bounds and approximations to several physical quantities, notably the torsional rigidity.

1. Preliminary definitions Let $D$ denote a bounded open convex plane domain and let $C$ denote the boundary of $D$. For each $p \in D$ and each $q \in C$, we denote by $h_{p q}$ the distance (regarded as positive) from $p$ to the supporting line to $D$ at $q$. The function $h_{p q}$ is single valued for almost all $q$.

Consider the family of functions defined by the integrals

$$
\oint_{c} h_{p q}^{\alpha} d s_{q},
$$

where $d s_{q}$ denotes an arc-element of $C$ at $q$.

For most values of the exponent $\alpha$, the integral in (1) depends upon $p$ as well as $D$. For the special cases $\alpha=0,1$ the integral (1) is independent of $p$. For $\alpha=0$ we obtain the length of $C$ which we denote by

$$
L(D)=L=\oint_{\sigma} d s_{q} .
$$

For $\alpha=1$, we obtain twice the area of $D$, which we denote by

$$
2 A(D)=2 A=\oint_{C} h_{p q} d s_{q} .
$$

In this paper we will be primarily concerned with the value $\alpha=-1$. For this case we employ the notation

$$
B_{p}(D)=B_{p}=\oint_{c} h_{p q}^{-1} d s_{q} .
$$

If $D$ is transformed by a similarity transformation (i.e. linear isogonal map) into $D^{*}$ and if $p$ is transformed into $p^{*}$ then 


$$
B_{p}(D)=B_{p} *\left(D^{*}\right) .
$$

Hence the set function $B(D)$ defined by

$$
B(D)=B=\inf _{p \in D} B_{p}(D)
$$

is invariant under a similarity transformation and thus depends on the shape but not the size of the convex domain. It is the set function referred to in the title.

2. Two analytic representations of $\boldsymbol{B}_{p}$. Consider a polar coordinate system with pole at $p$ and let $r$ and $\phi$ denote the polar coordinates of $q \in C$. The polar equation of $C$ is $r=r(\phi)$. Now

$$
d s_{q}^{2}=\left(r^{2}+r^{/ 2}\right) d \phi^{2}
$$

and by comparing two expressions for the area of an appropriate wedge with vertex at $p$ we obtain

$$
h_{p q} d s=r^{2} d \phi .
$$

Combining (7) and (8) we obtain

$$
\frac{d s_{q}}{h_{p q}}=\left(1+\frac{r^{/ 2}}{r^{2}}\right) d \phi
$$

This yields

$$
B_{p}=\int_{0}^{2 \pi}\left(1+\frac{r^{2}}{r^{2}}\right) d \phi .
$$

Another useful representation is for the case when the curve $C$ is described by parametric equations $x=x(t), y=y(t) ; 0 \leqq t \leqq T$. We adopt the convention that the domain $D$ lies in the $x, y$-plane, that $p$ corresponds to $x=0, y=0$. Also, since $C$ is convex, we may select our parameter $t$ so that $x^{\prime}(t), y^{\prime}(t)$ exist for almost all $t$ and so that $W(t)=$ $x(t) y^{\prime}(t)-x^{\prime}(t) y(t)>0$ for all $t$.

Then if there is a tangent line at $q=q(t)$ it has the equation ( $X$, $Y$ are coordinates of a point on the tangent line).

$$
x^{\prime}(t)\{Y-y(t)\}=y^{\prime}(t)\{X-x(t)\} .
$$

From the normal form of (11) we obtain

$$
h_{p q}=\frac{x(t) y^{\prime}(t)-x^{\prime}(t) y(t)}{\left[x^{\prime}(t)^{2}+y^{\prime}(t)^{2}\right]^{1 / 2}}=\frac{W(t)}{\left[x^{\prime}(t)^{2}+y^{\prime}(t)^{2}\right]^{1 / 2}} .
$$

The arc-element $d s_{q}$ can be expressed by 


$$
d s_{q}=\left[x^{\prime}(t)^{2}+y^{\prime}(t)^{2}\right]^{1 / 2} d t .
$$

Hence we obtain

$$
B_{n}=\int_{0}^{T} \frac{x^{\prime}(t)^{2}+y^{\prime}(t)^{2}}{W(t)} d t
$$

3. Inequalities involving $B_{p}$. We first show that of all convex domains, $D, B(D)$ is smallest for the circle. From (9) we obtain

$$
B_{p}(D)=2 \pi+\int_{0}^{2 \pi} \frac{r^{\prime}(\phi)^{2}}{r(\phi)^{2}} d \phi
$$

which implies

$$
B_{p} \geq 2 \pi .
$$

Since (13) is true for all $p$, we obtain

$$
B(D) \geqq 2 \pi
$$

We may strengthen (14) by applying the Schwarz Inequality to the functions $\sqrt{h_{p q}}$ and $1 / \sqrt{h_{p q}}$. We obtain

$$
\oint_{c} h_{p q}^{-1} d s_{q} \cdot \oint_{c} h_{p q} d s_{q} \geqq\left\{\oint_{c} d s_{q}\right\}^{2}
$$

Using the definitions in (2), (3) and (4) we obtain

$$
B_{p} \geqq \frac{L^{2}}{2 A}
$$

for all $p \in D$ and hence

$$
B \geqq \frac{L^{2}}{2 A} .
$$

By the classical isoperimetric theorem,

$$
L^{2} \geqq 4 \pi A
$$

with equality holding only for the circle.

Equality occurs in (15) and hence in (16) and (17) only when $\sqrt{h_{p q}}$ and $1 / \sqrt{h_{p q}}$ are proportional, i.e. when $h_{p q}=$ const, almost everywhere.

This is true only when $C$ consists of tangents to and arcs of a fixed circle whose center is at $p$. We call this class of curves circumscribable. Among the circumscribable polygons are the general triangle, the general rhombus, and the regular polygons. The fixed circle involved in the description of the domains for which $h_{p q}$ is constant almost everywhere, has the property that no larger circle can be contained in $\bar{D}$ (the closure 
of $D$ ). In general we define for an arbitrary domain $D$ a maximal inscribed circle as one of radius $\rho(D)$, which is contained in $\bar{D}$, where $\rho=\rho(D)$ is characterized by the following two properties:

(1) There exists in $\bar{D}$ a circle of radius $\rho$.

(2) There does not exist in $\bar{D}$ a circle of radius larger than $\rho$. The number $\rho(D)$ is unique but there may be several maximal inscribed circles as in the case when $D$ is a rectangle.

In those cases in which $D$ is convex and $B=L^{2} / 2 A$, ( $h_{p q}=$ constant almost everywhere), we have $h_{p q}=\rho(D)$ for the point $p$ at which $B_{p}=B=L / \rho$.

In all other cases ( $D$ is convex, $B>L^{2} / 2 A, h_{p q}$ not constant almost everywhere for any fixed $p$ ), let $p$ be a center of a maximal inscribed circle. Since $D$ is convex $h_{p q} \geqq \rho$ for all $q$ on $C$, and $h_{p q}>\rho$ for a set of positive measure on $C$. Hence

$$
\frac{L}{\rho}>B_{p}(D) \geqq B(D) .
$$

Combining the inequalities of the section we obtain

TheoRem 1. For all convex domains $L / \rho \geqq B \geqq L^{2} / 2 A \geqq 2 \pi$. Furthermore $L / \rho=B=L^{2} / 2 A \geqq 2 \pi$ for all circumscribable domains. For all other domains $L / \rho>B>L^{2} / 2 A>2 \pi$. The circle is the only domain for which $L / \rho=B=L^{2} / 2 A=2 \pi$.

As a consequence of Theorem 1, we have the following

COROLLARY. There exists a constant $M>0$ such that for any bounded convex domain $D$

$$
M \leqq \frac{B(D) \rho(D)}{L(D)} \leqq 1
$$

$M$ is of course independent of $D$.

The proof of the corollary depends upon the following geometric lemma which we state without proof. The proof of the lemma can be found in $[4$, pp. 509-510].

Lemma Let $D$ be a convex bounded domain and let $\bar{D}$ be the closure of $\bar{D}$. Then there exist closed rectangles $R_{1}, R_{2}$ with the following properties

(1) $R_{1} \subset \bar{D} \subset R_{2}$ 
(2) $R_{1}$ and $R_{2}$ are similar and similarly situated

( 3 ) $A\left(R_{2}\right) \leqq 9 A\left(R_{1}\right)$.

Proof of the Corollary. For an arbitrary rectangle we have $L \geqq 8 \rho$ and $A=\rho(L-4 \rho)$. Hence

$$
\frac{L \rho}{2 A}=\frac{L}{2 . L-4 \rho} \geqq \frac{1}{2} .
$$

Let $R_{1}, R_{2}$ be the two rectangles associated with $D$ in the lemma. Then

$$
\begin{aligned}
\frac{B(D) \rho(D)}{L(D)} & \geqq \frac{L(D) \rho(D)}{2 A(D)} \geqq \frac{L\left(R_{1}\right) \rho\left(R_{1}\right)}{2 A\left(R_{2}\right)} \\
& \geqq \frac{L\left(R_{1}\right) \rho\left(R_{1}\right)}{9.2 A\left(R_{1}\right)} \geqq \frac{1}{18}:
\end{aligned}
$$

Hence, using Theorem 1 again, we get $1 / 18 \leqq B \rho / L \leqq 1$ for all convex bounded domains.

4. Behavior of $\mathbf{B}_{p}$ near the boundary. At each point of a convex curve, there exist two one-sided tangents which are collinear (when extended) for all points with at most a countable number of exceptions.

In the present section we consider $h_{p q}$ to be always measured to the right hand tangent so that certain details of proof become easier. The values of $B_{p}$ and $B$ are not affected by this convention. By the righthand tangent we mean the one-sided tangent which points in the counterclockwise direction along the curve.

THeorem 2. As $p$ approaches the boundary curve $C, B_{p}$ becomes arbitrarily large. More precisely, if $q$ be any point of $C$, and $M a$ positive number, there exists a positive number $\delta$, such that $B_{\rho}>M$, for all $p \in D$ whose distance from $q$ is less then $\delta$.

Proof. We consider a Cartesian coordinate system whose origin is at $q$ and whose positive $x$-axis coincides with the right hand tangent at $q$. Hence $y>0$ for all points of $D$. There is a unique supporting line of $C$, which has slope one and whose $y$-intercept is negative. Let $t$ be the point of this line which belongs to $C$ and is closest to the origin at $q$. Let $q^{\prime}$ denote a variable point between $q$ and $t$ (such that $q, q^{\prime}, t$ occur in counterclockwise direction). Then

$$
B_{p}>\int_{q}^{\iota} \frac{d s_{q}^{\prime}}{h_{p q}^{\prime}} .
$$

The integral is taken along an arc of $C$. We denote by $M_{q^{\prime}}$, the slope 
of the right hand tangent at $q^{\prime}$. If the coordinates of $q$ are $(x, y)$ and those of a point on the right-hand tangent at $q^{\prime}$ are $(X, Y)$, the equation of tangent line is

$$
Y-y=M_{q^{\prime}}(X-x) .
$$

Let $p$ be a point of $D$ with coordinates $(\lambda \delta, \mu \delta)$ where $\delta>0, \mu>0, \lambda \geqq 0$, $\lambda^{2}+\mu^{2} \leqq 1$.

From (22) we obtain

$$
h_{p q^{\prime}}=\frac{\mu \delta-y-M_{q^{\prime}} \lambda \delta+M_{q^{\prime}} x}{1+M_{q^{\prime}}{ }^{2}} .
$$

Also

$$
d s_{q^{\prime}}=\left(1+M_{q^{\prime}}{ }^{2}\right) d x .
$$

Combining these expressions with (21) we obtain

$$
B_{p}>\int_{0}^{a} \frac{1+M_{q^{\prime}}{ }^{2}}{\mu \delta-y-M_{q^{\prime}} \lambda \delta+M_{q^{\prime}} x} d x
$$

where $a$ is the abscissa of the point $t$. Since $0 \leqq M_{q^{\prime}} \leqq 1$

$$
B_{p}>\int_{0}^{a} \frac{d x}{\delta+x}=\log \frac{a+\delta}{\delta}>\log \frac{a}{\delta} .
$$

Hence, given $M>0$, we can find $\delta^{\prime}>0$ such that, for all $p \in D$ for which dist. $(p, q) \leqq \delta^{\prime}$ and which are in the closed first quadrant (of the cooddinate system described above) we have $B_{p}>M$.

Similarly (by considering a coordinate system with origin at $q$, whose negative $x$-axis coincides with the left hand tangent at $q$, and measuring $h_{p q}$ to the left hand tangent at $q$, etc.) we can find $\delta^{\prime \prime}>0$ such that for all $p \in D$ for which dist. $(p, q) \leqq \delta^{\prime \prime}$ and which are in the closed second quadrant of this new coordinate system we have $B_{p}>M$.

The two closed quadrants share a boundary if $q$ is a point of tangency and an entire angle if $q$ is not a point of tangency since $D$ is convex. Hence if $\delta=\min \left(\delta^{\prime}, \delta^{\prime \prime}\right)$ and $p \in D$ with dist. $(p, q) \leqq \delta$ we obtain $B_{p}>M$.

Corollary The function $B_{p}$ assumes its minimum value for some $P$ in $D$.

Proof $1 / B_{p}>0$ in $D$ and $1 / B_{p} \rightarrow 0$ as $p \rightarrow C$. Also $1 / B_{p}$ is continuous in $D$. Hence $1 / B_{p}$ attains its maximum value in $D$.

To show the continuity of $B_{p}$ and hence of $1 / B_{p}\left(B_{p} \neq 0\right)$ we proceed as follows. Let $d\left(p_{1}, p_{2}\right)$ denote the distance from $p_{1}$ to $p_{2}$, and let 
$d(p, C)$ denote $\inf _{q \in C} d(p, q)$. Fix $p \in D$. Consider all $p^{\prime}$ such that $d\left(p, p^{\prime}\right) \leqq \lambda d(p, C)$ where $\lambda$ is a fixed number in $0<\lambda<1$. Then

$$
\begin{aligned}
& \left|h_{p q}-h_{p^{\prime} q}\right| \leqq d\left(p, p^{\prime}\right) . \\
& \left|\frac{1}{h_{p q}}-\frac{1}{h_{p q}},\right| \leqq \frac{d\left(p, p^{\prime}\right)}{(1-\lambda) d^{2}(p, C)} .
\end{aligned}
$$

Hence

$$
\left|B_{p}-B_{p^{\prime}}\right| \leqq \frac{L}{(1-\lambda) d^{\prime}(p, C)} \cdot d\left(p, p^{\prime}\right)
$$

Hence as $p^{\prime} \rightarrow p ; B_{p^{\prime}} \rightarrow B_{p}$.

5. The convexity of $\mathbf{B}_{p}$. We consider a Cartesian coordinate system with origin 0 at some interior point of $D$. Let $\theta=\theta_{q}$ be the angle between the positive direction of the $x$-axis and the outward normal at $q$. If there is no tangent at $q$ we use (for definiteness) the normal to the right-hand tangent to $C$ at $q$. The equation of the tangent line at $q$ is

$$
X \cos \theta_{q}+Y \sin \theta_{q}=h_{0 q} .
$$

In (24) $X, Y$ are coordinates of a point on the tangent line, and $h_{\mathrm{cq}}$ is the value of $h_{p q}$ at the origin. If the coordinates of an arbitrary point, $p \in D$ are $(x, y)$ then from (24) which is in the normal form, we obtain

$$
h_{p q}=h_{0 q}-x \cos \theta_{q}-y \sin \theta_{q} .
$$

If we differentiate (25) we obtain

$$
\frac{\partial h_{p q}}{\partial x}=-\cos \theta_{q}
$$

and

$$
\frac{\partial h_{p q}}{\partial y}=-\sin \theta_{q}
$$

From (4), (26) and (27) we obtain the various derivatives of $B_{p}$. They are

$$
\begin{aligned}
& \frac{\partial B_{p}}{\partial x}=\oint_{c} \frac{\cos \theta_{q}}{h^{2}{ }_{p q}} d s_{q}, \\
& \frac{\partial B_{p}}{\partial y}=\oint_{c} \frac{\sin \theta_{q}}{h^{2}{ }_{p q}} d s_{q},
\end{aligned}
$$




$$
\begin{aligned}
\partial^{2} B_{p} & =2 \oint_{c} \frac{\cos ^{2} \theta_{q}}{h_{p q}^{3}} d s_{q}, \\
\frac{\partial^{2} B_{p}}{\partial y^{2}} & =2 \oint_{c} \frac{\sin ^{2} \theta_{q}}{h_{p q}^{3}} d s_{q},
\end{aligned}
$$

and

$$
\frac{\partial^{2} B_{p}}{\partial x \partial y}=2 \oint_{c} \frac{\sin \theta_{q} \cos \theta_{q}}{h_{p q}^{3}} d s_{q}
$$

From (30) and (31) we obtain

$$
\frac{\partial^{2} B_{p}}{\partial x^{2}}+\frac{\partial^{2} B_{p_{-}}}{\partial y^{2}}=2 \oint_{c} \frac{d s_{q}}{h_{p q}^{3}}>0
$$

Hence $B_{p}$ is a strictly subharmonic function of $p \in D$. We now prove that $B_{p}$ is also convex in $D$.

THeOREM 3. The function $B_{p}$ is strictly convex in $D$.

Proof Consider the quadratic form

$$
Q(u, v)=\frac{\partial^{2} B_{p}}{\partial x^{2}} u^{2}+2 \frac{\partial^{2} B_{p}}{\partial x \partial y} u v+\frac{\partial^{2} B_{p}}{\partial y^{2}} v^{2} .
$$

From (30), (31), and (32) we obtain

$$
Q(u, v)=2 \oint_{c} \frac{\left(u \cos \theta_{q}+v \sin \theta_{q}\right)^{2}}{h_{p q}^{3}} d S_{q} .
$$

A sufficient condition [1; Th. 99] that $B_{p}$ be strictly convex in $D$ is that $Q(u, v)>0$ for all $(u, v) \neq(0,0)$ and all $p \in D$. Clearly from (35) $Q(u, v) \geqq 0$. Let us assume that for some $\left(u_{0}, v_{0}\right) \neq(0,0), Q\left(u_{0}, v_{0}\right)=0$. Then it would follow that

$$
u_{0} \cos \theta_{q}+v_{0} \sin \theta_{q}=0
$$

for almost all $q$.

Hence for almost all $q, \theta_{q}$ would be limited to two distinct values in the interval $0 \leqq \theta_{q}<2 \pi$. These two values would differ by $\pi$. Hence if we except a set of measure zero from the boundary of $D$, all of the supporting lines of $D$ would be parallel and $D$ would have but two supporting lines. This situation is possible for an infinite convex domain; i.e. the infinite strip between two parallel lines. However it is not possible for bounded convex domains. For let $L$ and $L^{*}$ be the two lines. A supporting line of a convex domain at a point of its boundary must contain this point. However there are two ares of positive length 
between $L$ and $L^{*}$. The lines $L$ and $L^{*}$ cannot be supporting lines to any point of there arcs. Hence for a bounded convex domain, the numbers $\left(u_{0}, v_{0}\right) \neq(0,0)$ cannot exist such that $Q\left(u_{0}, v_{0}\right)=0$ and $B_{p}$ is strictly convex.

An important corollary of Theorem 4.3 is that there is but one point of $D$, at which $B_{p}$ assumes its minimum value, $B(D)$. For if there were two points $p^{\prime}$ and $p^{\prime \prime} \in D$ such that $B(D)=B_{p^{\prime}}=B_{p^{\prime \prime}}$, it would follow from the strict convexity [4, th. 98] of $B_{p}$ that for all $p$ on the segment joining $p^{\prime}$ and $p^{\prime \prime}, B_{p}$ would be less than $B(D)$. This is impossible so we obtain the following.

THEOREM 4. The Equation $B_{p}(D)=B(D)$ has one and only one solution $p=p_{0}$ in $D$. Furthermore $B_{p}$ has no relative extrema except at $p_{0}$.

Proof. The existence and uniqueness of $\mathrm{p}_{\mathrm{v}}$ has already been demonstrated. As for the existence of other extrema, there can be no maxima or saddle points because of (33) and (34). As for another relative minimum say at a point $p^{*}$, there would be points $p$ arbitrarily close to $p^{*}$ on the segment joining $p^{*}$ to $p_{0}$ for which the value of $B_{p}$ would be less than $B_{p}{ }^{*}$.

A consequence of Theorem 4 is that the usual necessary conditions for relative extrema are necessary and sufficient conditions for the unique absolute minimum value of $B_{p}$.

Theorem 4 has the geometric interpretation that a convex surface can only have a single horizontal supporting plane.

Theorem 4 also facilitates the determination of $p_{0}$ and hence of $B(D)$ in the case of a domain with symmetry.

Corollary 1. If $D$ possesses a line of symmetry, $B_{p}(D)$ assumes its minimum upon that line.

CoRollary 2. If $D$ possesses two lines of symmetry, intersecting at $p_{0}$, then $B(D)=B_{p 0}(D)$.

Corollary 3. If $D$ possesses a center of rotation (in the sense that $D$ is invariant under a rotation of $2 \pi / n$ radian about this point, where $n>1$ is an integer) then $B_{p}$ assumes its minimum at this point.

6. Steiner symmetrization and B. By Steiner symmetrization of a plane domain $D$ with respect to a fixed straight line $l$, we mean the following: $D$ is transformed into the domain $D^{*}$ which is characterized by the properties; 
(a) $D^{*}$ is symmetric about the line $l$.

(b) The intersections of any line perpendicular to $l$ with $D$ and $D_{*}$ are of the same length.

THEOREM 5. Let $D$ be a bounded convex domain with boundary $C$. Let $D$ be transformed by Steiner symmetrization about a line l, into the domain $D^{*}$ and let $C^{*}$ be the boundary of $D^{*}$. Then $D^{*}$ is convex and $B(D) \geqq B\left(D^{*}\right)$. Further if the line $l$ is not parallel to an axis of symmetry of $D$ then $B(D)>B\left(D^{*}\right)$.

Proof. Since the sum of convex functions is convex, it is clear that $D^{*}$ is convex and hence $B\left(D^{*}\right)$ is defined. Let us consider a coordinate system with origin at the point at which $B_{p}(D)$ has its minimum. Let the line with respect to which we symmetrize $D$ be the $x$-axis. Let $a \leqq x \leqq b$ be the projection of $\bar{D}$ upon the $x$-axis. We denote by $\lambda_{a}$ and $\lambda_{b}$ the lengths of those portions of $C$ for which $x=a$ and $x=b$ respectively. The two portions of $C$ which lie between the supporting lines $x=a$ and $x=b$ can be represented by the two single valued continuous functions,

$$
y=u(x), y=v(x) \quad a<x<b,
$$

with

$$
u(x)>v(x) .
$$

We use (12) with $x(t)=t$ and $y(t)=u(t)$ or $v(t)$ according as to whether we consider the upper or lower branch of $C$. Then if we remember that the positive direction of $x$ (or $t$ ) coincides with the clockwise direction for $u(x)$ and the counter-clockwise direction for $v(x)$ we obtain

$$
B=B_{0}=\frac{\lambda_{b}}{b}-\frac{\lambda_{a}}{a}+\int_{a}^{b}\left[\frac{1+u^{\prime}(t)^{2}}{u(t)-t u^{\prime}(t)}+\frac{1+v^{\prime}(t)^{2}}{t v^{\prime}(t)-v(t)}\right] d t .
$$

If we symmetrize $D$ with respect to the $x$-axis we may denote the two branches of the boundary of $D^{*}$ by

$$
y= \pm w(x)= \pm \frac{1}{2}(u(x)-v(x) .)
$$

Then

$$
B_{0}^{*}=\frac{\lambda_{b}}{b}-\frac{\lambda_{a}}{a}+2 \int_{a}^{b} \frac{1+w^{\prime 2}(t)}{w(t)-t w^{\prime}(t)} d t \geqq B^{*}
$$

If we introduce the abbreviations

$$
U_{1}^{2}=1+u^{\prime}(t)^{2}
$$




$$
\begin{array}{rlr}
U_{2} & =u(t)-t u^{\prime}(t)>0 & \\
V_{1}^{2} & =1+v^{\prime}(t)^{2}, & \\
V_{2} & =t v^{\prime}(t)-v(t)>0 & \\
Z & =1-u_{1}(t) v^{\prime}(t) &
\end{array}
$$

we obtain

$$
B(D)=B_{0}(D)=\frac{\lambda_{b}}{b}-\frac{\lambda_{a}}{a}+\int_{a}^{b}\left(\frac{U_{1}^{2}}{U_{2}}+\frac{V_{1}^{2}}{V_{2}}\right) d t
$$

and

$$
B_{0}\left(D^{*}\right)=\frac{\lambda_{b}}{b}-\frac{\lambda_{a}}{a}+\int_{a}^{b}\left(\frac{U_{1}^{2}+V_{1}^{2}+2 Z}{U_{2}+V_{2}}\right) d t
$$

Hence

$$
B_{0}(D)-B_{0}\left(D^{*}\right)=\int_{a}^{b}\left[\frac{U_{1}^{2}}{U_{2}}+\frac{V_{1}^{2}}{V_{2}}-\frac{U_{1}^{2}+V_{1}^{2}+2 Z}{U_{2}+V_{2}}\right] d t
$$

We shall next prove that the integrand is always non-negative. First $U_{1}^{2} V_{1}^{2}=Z^{2}+\left\{u^{\prime}(t)^{2}+v^{\prime}(t)^{2}\right\}$ and therefore

$$
U_{1}^{2} V_{1}^{2} \geqq Z^{2},
$$

which implies

$$
\left(U_{1} V_{1}-Z\right) \geqq 0 .
$$

Hence we obtain successively,

$$
\begin{gathered}
\left(U_{2} V_{1}-U_{1} V_{2}\right)^{2}+2 U_{2} V_{2}\left(U_{1} V_{1}-Z\right) \geqq 0, \\
\left(U_{1}^{2} V_{2}+V_{1}^{2} U_{2}\right)\left(U_{2}+V_{2}\right) \geqq U_{2} V_{2}\left(U_{1}^{2}+V_{1}^{2}+2 Z\right),
\end{gathered}
$$

and

$$
\frac{U_{1}^{2}}{U_{2}}+\frac{V_{1}^{2}}{V_{2}} \geqq \frac{U_{1}^{2}+V_{1}^{2}+2 Z}{U_{2}+V_{2}} .
$$

Hence from (38) we finally obtain

$$
B_{0}\left(D^{*}\right) \leqq B_{0}(D)
$$

but $B_{0}\left(D^{*}\right) \geqq B\left(D^{*}\right)$ and $B_{0}(D)=B(D)$ and thus

$$
B(D) \geqq B\left(D^{*}\right) \text {. }
$$

Equality in (38) and hence in (43) cannot occur unless it occurs almost everywhere in (39). But this would imply that $u^{\prime}(t)+v^{\prime}(t)=0$ almost 
everywhere and since $u$ and $v$ are continuous that $u(t)+v(t)$ is constant. But this can only happen if $D$ has a line of symmetry parallel to the $x$-axis.

7. Symmetrical domains. In this section we consider convex domains, $D$, which possess the symmetries of the rectangle. Such domains possess two axes of symmetry which are at right angles to each other. We establish a rectangular coordinate system in which the axes of symmetry correspond to the lines $y=x$ and $y=-x$. The origin is then the center of symmetry. Let $D^{*}$ be the domain obtained from $D$ by the affine transformation.

$$
X=\mu x, \quad Y=y .
$$

Then $D^{*}$ will also be convex and have the origin as a center of symmetry. For both $D$ and $D^{*}$ we must compute $B$ at the origin (see Corollary 3, Theorem 4). The Boundary $C$ of $D$ can be expressed parametrically in the form $x=x(t), y=y(t) ; 0 \leqq t \leqq T$. We select the parameter, so that the Wronskian, $W(t)=x(t) y^{\prime}(t)-y(t) x^{\prime}(t)$ is always positive. Furthermore we select the parameter so that $t=0$ and $t=T$ correspond to the intersection of $C$ with the line $y=x$ in the first quadrant. Finally we select the parameter so that symmetrical parts of the curve correspond to equal intervals on the $t$-axis (for example, we may take $t$ as arc length). Then the symmetry conditions can be expressed analytically by

$$
x(T-t)=y(t), y(T-t)=x(t), \quad 0 \leqq t \leqq T .
$$

and

$$
x\left(\frac{T}{2}+t\right)=-x(t), y\left(\frac{T}{2}+t\right)=-y(t), \quad 0 \leqq t \leqq \frac{T}{2} .
$$

THEOREM 6. In the notation of the present section we have

$$
B\left(D^{*}\right)=B(D)\left\{\frac{\mu+\mu^{-1}}{2}\right\} \text {. }
$$

Proof. By (12) we have

$$
B(D)=B_{0}(D)=\int_{0}^{t} \frac{x^{\prime}(t)^{2}+y^{\prime}(t)^{2}}{x(t) y^{\prime}(t)-x^{\prime}(t) y(t)} d t .
$$

[By $B_{0}$ we mean $B_{p}$ at the origin]. Also

$$
B\left(D^{*}\right)=\int_{0}^{T} \frac{\mu^{2} x^{\prime}(t)^{2}+y^{\prime}(t)^{2}}{\mu W(t)} d t .
$$


Using (45) we obtain

$$
\int_{T / 2}^{T} \frac{\mu^{2} x^{\prime}(t)^{2}+y^{\prime}(t)^{2}}{\mu W(t)} d t=\int_{0}^{T / 2} \frac{x^{\prime}(t)^{2}+\mu^{2} y^{\prime}(t)^{2}}{\mu W(t)} d t
$$

Hence

$$
\begin{aligned}
& B\left(D^{*}\right)=\int_{0}^{T / 2} \frac{\mu^{2} x^{\prime}(t)^{2}+y^{\prime}(t)^{2}}{\mu W(t)} d t \\
& +\int_{T / 2}^{T} \frac{x^{\prime}(t)^{2}+\mu^{2} y^{\prime}(t)^{2}}{\mu W(t)} d t \\
& =\left(\mu+\mu^{-1}\right) \int_{0}^{T / 2} \frac{x^{\prime}(t)^{2}+y^{\prime}(t)^{2}}{W(t)} d t .
\end{aligned}
$$

From (46) and (47), we have

$$
B(D)=2 \int_{0}^{T / 2} \frac{x^{\prime}(t)^{2}+y^{\prime}(t)^{2}}{W(t)} d t
$$

From (50) and (51) we obtain

$$
B\left(D^{*}\right)=B(D)^{\mu+\mu^{-1}},
$$

which completes the proof of the theorem.

8. Some special domains. In this section we compute $B(D)$ explicitly for several domains, using the methods developed in the earlier sections. The results are summarized in table form in [4].

8.1 Circumscribable domains. Theorem 1 includes the statement that for circumscribable domains, $B=L / \rho=L^{2} / 2 A$. The most important special cases involved are the regular polygons, the general triangle, the rhombus and, of course, the circle. We list the results for these domains without details.

Domain $D$

(53)

$$
B(D)
$$

$2 \pi$

$$
\text { Regular } N \text {-gon. } \quad 2 N \tan \frac{\pi}{N} \text {. }
$$$$
2 s \sqrt{\frac{s}{(s-a)(s-b)(s-c)}} .
$$ 
(56) Rhombus of angle $\delta . \quad \frac{8}{\sin \delta}$.

From either (54) or (56) we obtain for the equilateral triangle, the value $B=6 \sqrt{3}$.

8.2 The domain. $|x|^{n}+|y|^{n}<1$. The domain $|x|^{n}+|y|^{n}<1$ is convex for $n \geqq 1$ and has the origin as a center of symmetry. Hence $B=B_{0}$ ( $B_{p}$ at the origin). It is most convenient to denote the boundary of $D$ parametrically in the form

$$
x=\left(\cos ^{2} t\right)^{n} ; y=\left(\sin ^{2} t\right)^{n} \quad 0 \leqq t \leqq 2 \pi .
$$

Then letting $\lambda=2 / n$ and taking advantage of symmetry, we obtain from (12) that

$$
\begin{aligned}
B & =8 \lambda \int_{0}^{\pi / 2} \cos ^{(\lambda-1)} t \sin ^{3-\lambda} t d t \\
& =\frac{8}{n} \Gamma\left(\frac{1}{n}\right) \Gamma\left(2-\frac{1}{n}\right) .
\end{aligned}
$$

For $n>1$. We can simplify using the relation $\Gamma(z) \Gamma(1-z)=\pi / \sin \pi z$ to obtain,

$$
B=\frac{8(n-1)}{n^{2}} \cdot \frac{\pi}{\sin \frac{\pi}{n}}, \quad n>1
$$

As special or limiting cases of $(57)$ we have the circle $(n=2)$ and the square $\left(n \rightarrow \infty\right.$ and $\left.n \rightarrow 1^{+}\right)$.

8.3 Application of Theorem 6. We mention just two applications. If we apply the affine transformation $X=a x / b, Y=y$ to the circle $x^{2}+y^{2}=b^{2}$, we obtain the ellipse $X^{2} / a^{2}+Y^{2} / b^{2}=1$. Then by Theorem 6 we obtain for this ellipse

$$
B=\pi\left\{\frac{a}{b}+\frac{b}{a}\right\} .
$$

For the square we may obtain from (54) or (56) or (57) the value

$$
B=8 \text {. }
$$

If we place the square so that its sides are parallel to the coordinate axis, the affine transformation (44) results in a rectangle. If the sides of the rectangle are in the proportion $a: b$, Theorem 6 yields 


$$
B=4\left\{\frac{a}{b}+\frac{b}{a}\right\}
$$

If we place the square so that the diagonal coincide with the axis, the affine transformation results in a rhombus. Thus using Theorem 6 we may obtain (56) from (59) as well as (59) from (56).

8.4 Convex circular sectors. We consider domains $D_{\lambda}$ defined in polar coordinates by $0<\rho<1 ;-\lambda \pi<\phi<\lambda \pi$. For convexity we restrict $\lambda$ to the range $0<\lambda \leqq 1 / 2$. The polar axis, $\phi=0$ is a line of symmetry for the sector and by Corollary 1 of Theorem 4.4, $\mathrm{B}_{p}(D \lambda)$ assumes its minimum value $B\left(D_{\lambda}\right)=B^{(\lambda)}$ on this line. Let $(u, 0)$ be the coordinates of any point $p$ on this line of symmetry, $0<u<1$. If we denote $B_{p}\left(D_{\lambda}\right)$ by $B_{u}\left(D_{\lambda}\right)$ we obtain

$$
\begin{aligned}
B_{u}\left(D_{\lambda}\right) & =\frac{2}{u \sin \lambda \pi}+2 \int_{0}^{\lambda \pi} 1-u \cos t \\
& =\frac{2}{u \sin \lambda \pi}+\frac{4}{\sqrt{ } 1-u^{2}} \arctan \{\sqrt{1+u} \\
1-u & \left.\tan \frac{\lambda \pi}{2}\right\} .
\end{aligned}
$$

It is difficult to express $B^{(\lambda)}$ as an explicit function of $\lambda$, but for a particular $\lambda$, the computation can be carried out numerically.

In the case of the semi-circle $\lambda=1 / 2$ and (61) becomes

$$
B_{u}\left(D_{1 / 2}\right)=\frac{2}{u}+\frac{4}{V 1-u^{2}} \arctan \sqrt{\frac{1+u}{1-u}} .
$$

If we set $y^{2}=1+u / 1-u$ and express $\arctan y$ as the sum of an odd and an even function of $u$, we obtain arctan $y=\pi / 4+1 / 2 \arctan$ $\left(y^{2}-1\right) / 2 y$. But $\left(y^{2}-1\right) / 2 y=u / \sqrt{ } 1-u^{2}$ and hence $\arctan \left[\left(y^{2}-1\right) / 2 y\right]=$ $\arcsin u$.

Finally we obtain for the semi-circle

$$
B_{u}\left(D_{1 / 2}\right)=\frac{2}{u}+\frac{2 \arcsin u+\pi}{\sqrt{1-u^{2}}} .
$$

The derivative of the right side has a unique zero in the interval. We can compute this zero, $u_{0}$, by Newton's method and then compute $B u_{0}\left(D_{1 / 2}\right)$. This yields $B^{(1 / 2)}=8.7915$.

Another special case of interest is the 'narrow' sector corresponding to 'small' values of $\lambda$. We use Theorem 1 and first obtain expressions for $L, A$, and $\rho$.

$$
L=2+2 \lambda \pi
$$




$$
\begin{aligned}
& A=\lambda \pi, \\
& \rho=\frac{\sin \lambda \pi}{1+\sin \lambda \pi} .
\end{aligned}
$$

The last expression is obtained by noting that the center of the maximum inscribed circle lies on the axis of symmetry and that the circle itself is tangent to the circular arc of the sector. Hence its center is at $(1-\rho, 0)$ and $\rho$ satisfies $\rho=(1-\rho) \sin \lambda \pi$. Theorem 1 states that $L / \rho>B>L^{2} / 2 A$, or that

$$
2 \frac{(1+\lambda \pi)(1+\sin \lambda \pi)}{\sin \lambda \pi}>B^{(\lambda)}>\frac{2}{\lambda \pi}(1+\lambda \pi)^{2} .
$$

If we expand is powers of $\lambda$ we obtain

$$
\frac{2}{\lambda \pi}+4+\frac{7}{3} \lambda \pi+O\left(\lambda^{2}\right)>B^{(\lambda)}>\frac{2}{\lambda \pi}+4+2 \lambda \pi+O\left(\lambda^{2}\right)
$$

Hence

$$
B^{(\lambda)}=\frac{2}{\lambda \pi}+4+O(\lambda)
$$

By a more involved analysis using (61) and (64) we can obtain

$$
B^{(\lambda)}=\frac{2}{\lambda \pi}+4+\frac{9}{4} \lambda \pi+O\left(\lambda^{2}\right) .
$$

9. An analogy involving $B_{p}$. Let $D$ be a simply-connected domain in the plane, and let $p \in D$. If we map $D$ conformally and in a oneto-one manner onto a circle whose center is the image of $p$, and for which the derivative of the mapping function has modulus one at $p$, then the radius of the circle is uniquely determined. We call this radius the inner conformal radius of $D$ with respect to $p$, and denote it by $r_{p}(D)$. As $p$ approaches the boundary of $D, r_{p}(D)$ approaches zero. This suggests an analogy between $1 / r_{p}$ and $B_{p}$. To strengthen this analogy we consider the function $2 A / r_{p}^{2}$. For the essential properties of $r_{p}(D)$ which we use, reference may be made to [3, vol. 2, chapter 4, probs. $110,112,124,125]$ and to [2]. We now restrict $D$ to be a convex bounded domain and denote by $Q_{p}=Q_{p}(D)$ either of the following domain functions.

$$
\text { (1) } B_{p}(D), \quad \text { (2) } \frac{2 A(D)}{r_{p}^{2}(D)}
$$

We define $Q(D)=Q$ as $\sup _{p \in D} Q_{p}(D)$. Then we can assert the following : 
I. For fixed $D, Q_{p}$ becomes infinite as $p$ approaches the boundary of $D$.

II. For all $D, Q_{p} \geqq 2 \pi$, with equality holding only for the circle and $p$ at the center of the circle.

III. $Q_{p}(D)$ assumes the value $Q(D)$ for precisely one $p \in D$.

IV. $\rho(D) Q(D) / L(D)$ lies between fixed positive bounds (independent of $D$ ) for all $D$.

V. Steiner symmetrization cannot increase $Q(D)$.

\section{REFERENCES}

1. G. H. Hardy, J. E. Littlewood, and G. Pólya, Inequalities.

2. H. R. Haegi, Extremalprobleme und Ungleichungen konformer Gebietsgrössen, Comp. Math. 8 (1950,) 81-111.

3. G. Pólya, and G. Szegö, Aufgaben and Lehrsätze aus der Analysis.

4. G. Pólya, and G. Szegö, Isoperimetric inequalities in mathematical physics.

RADIATION LABORATORY

The JoHN Hopkins UNIVERSITY 



\section{PACIFIC JOURNAL OF MATHEMATICS}

\section{EDITORS}

David Gilbarg

Stanford University

Stanford, California

R. A. Beaumont

University of Washington

Seattle 5, Washington

\author{
A. L. Whiteman
}

University of Southern California

Los Angeles 7, California

E. G. Straus

University of California

Los Angeles 24, California

\section{ASSOCIATE EDITORS}

\author{
E. F. BECKENBACH \\ C. E. BURGESS \\ M. HALL \\ E. HEWITT
}

\author{
A. HORN \\ V. GANAPATHY IYER \\ R. D. JAMES \\ M. S. KNEBELMAN
}

L. NACHBIN
I. NIVEN
T. G. OSTROM
H. L. ROYDEN

M. M. SCHIFFER

G. SZEKERES

F. WOLF

K. YOSIDA

\section{SUPPORTING INSTITUTIONS}

\author{
UNIVERSITY OF BRITISH COLUMBIA \\ CALIFORNIA INSTITUTE OF TECHNOLOGY \\ UNIVERSITY OF CALIFORNIA \\ MONTANA STATE UNIVERSITY \\ UNIVERSITY OF NEVADA \\ OREGON STATE COLLEGE \\ UNIVERSITY OF OREGON \\ UNIVERSITY OF SOUTHERN CALIFORNIA
}

\author{
STANFORD UNIVERSITY \\ UNIVERSITY OF UTAH \\ WASHINGTON STATE COLLEGE \\ UNIVERSITY OF WASHINGTON \\ AMERICAN MATHEMATICAL SOCIETY \\ CALIFORNIA RESEARCH CORPORATION \\ HUGHES AIRCRAFT COMPANY \\ THE RAMO-WOOLDRIDGE CORPORATION
}

Mathematical papers intended for publication in the Pacific Journal of Mathematics should be typewritten (double spaced), and the author should keep a complete copy. Manuscripts may be sent to any of the editors. All other communications to the editors should be addressed to the managing editor, E. G. Straus at the University of California, Los Angeles 24, California.

50 reprints per author of each article are furnished free of charge; additional copies may be obtained at cost in multiples of 50 .

The Pacific Journal of Mathematics is published quarterly, in March, June, September, and December. The price per volume (4 numbers) is $\$ 12.00$; single issues, $\$ 3.50$. Back numbers are available. Special price to individual faculty members of supporting institutions and to individual members of the American Mathematical Society: $\$ 4.00$ per volume; single issues, $\$ 1.25$.

Subscriptions, orders for back numbers, and changes of address should be sent to Pacific Journal of Mathematics, 2120 Oxford Street, Berkeley 4, California.

Printed at Kokusai Bunken Insatsusha (International Academic Printing Co., Ltd.), No. 6, 2-chome, Fujimi-cho, Chiyoda-ku, Tokyo, Japan.

\section{PUBLISHED BY PACIFIC JOURNAL OF MATHEMATICS, A NON-PROFIT CORPORATION}

The Supporting Institutions listed above contribute to the cost of publication of this Journal, but they are not owners or publishers and have no responsibility for its content or policies. 


\section{Pacific Journal of Mathematics}

\section{Vol. 8, No. 3 \\ May, 1958}

Michael Israel Aissen, A set function defined for convex plane domaines... . 383

Robert Ellis, Distal transformation groups ................... 401

Ciprian Foias, On a commutative extension of a commutative Banach algebra ....................................... 407

Jerry William Gaddum, Linear inequalities and quadratic forms ......... 411

Allen A. Goldstein and Elliott Ward Cheney, Jr., A finite algorithm for the solution of consistent linear equations and inequalities and for the Tchebycheff approximation of inconsistent linear equations...........

William L. Hart and T. S. Motzkin, Proof of the fundamental theorem on implicit functions by use of composite gradient corrections .......... 429

Henry Berge Helson, Conjugate series and a theorem of Paley .......... 437

Wu-Chung Hsiang, Abelian groups characterized by their independent subsets....................................... 447

John W. Lamperti, On the isometries of certain function-spaces ........ 459

Karel DeLeeuw and Walter Rudin, Extreme points and extremum problems

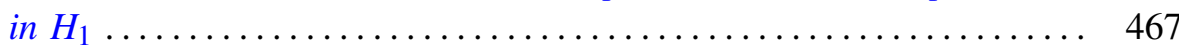

Eugene Lukacs, Some extensions of a theorem of Marcinkiewicz........ 487

George W. Mackey, Multiplicity free representations of finite groups ..... 503

Eben Matlis, Injective modules over Noetherian rings ............. 511

John William Neuberger, Continuous products and nonlinear integral equations

Lawrence Edward Payne and Hans F. Weinberger, New bounds for solutions of second order elliptic partial differential equations...

William T. Reid, A Prüfer transformation for differential systems ........ 575

Howard L. Rolf, The free lattice generated by a set of chains ...

K. M. Saksena, Inversion and representation theorems for a generalized

Laplace integral....................................... 597

Daniel Shanks, Two theorems of Gauss......................... 609

Paul Slepian, On the Lebesgue area of a doubled map ............... 613

Otto Szász and Nelson Paul Yeardley, Jr., The representation of an analytic function by general Laguerre series . ..................... 621

Alan C. Woods, On two-dimensional convex bodies ................. 635 